\title{
DOE Final Scientific/Technical Report
}

DOE Award Number: DE-FG02.-07ER46369

\section{Recipient: Arizona Board of Reagents}

\section{Title: Multiscale Simulation of Thermo-Mechanical Processes in Irradiated Fission- Reactor Materials}

\author{
Recipient Project Director: Louis Rene Corrales
}

\section{Executive Summary}

This research characterized, by atomic level molecular dynamics (MD) simulations, the early stage aggregation of the fission product xenon in a model uranium oxide nuclear fuel matrix that contributes to the long term bubble formation mechanisms that compromise the efficiency and lifetime of nuclear fuels. These atomic level computer simulations disclosed a previously unknown pre-coarsening phenomenon in which the clustering of xenon is driven by vacancy diffusion. These key findings are to be included in higher level simulations of nuclear fuel thermo-mechanical processes that model irradiation effects within nuclear fuels. Previous multiscale models did not include these early time pre-coarsened clusters that occur in the nanosecond regime and therefore will lead to a better understanding of bubble formation in nuclear fuels.

\section{Project Goal and Description}

Our goal was to train a student in large scale atomic level MD simulation methods to disclose the role of early time dynamics (e.g. in the nanosecond time scale regime) of xenon gas migration within a model uranium oxide fuel that had undergone irradiation damage. The student was first introduced to damage formation by studying how primary knock-on atom displacement energies lead to point defect formation and different types of point defect populations depending on angles and energy of displacement. The model uranium oxide fuel was a polycrystalline sample containing grain boundaries and using recently developed interaction potentials that capture the appropriate physics for defect formation at high temperatures. Next the student undertook the task of carrying out displacement cascade simulations in perfect crystals, crystals with varying populations of Schottky defects, and in crystals containing xenon gas within the Schottky defects. Finally, the student undertook the clustering study under reactor-like conditions of high temperature and by varying the amount of damage and fission gas products. This was done by starting from a perfect crystalline form of urania, introducing damage by randomly creating Schottky defects with the crystalline matrix, and then randomly distributing fission gas atoms within this damage matrix. Vacancy assisted migration was expected to play a role, however, details of the role of the grain boundaries and the population of Schottky defects needed to observe migration and aggregation was previously unknown. Results revealed that xenon atom clustering was driven by vacancy assisted migration and occurred at time scales shorter than those required to observe grain boundary migration. This result was surprising as experiment had previously revealed that xenon bubble formation is driven by grain boundary sweeping. Thus, these findings will play an essential role in multiscale model. 

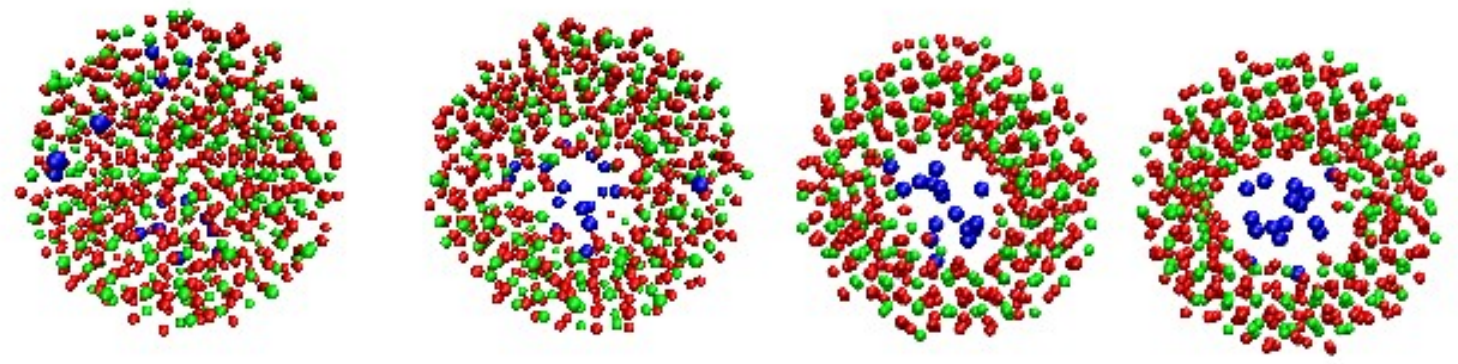

Figure. Vacancy-assisted migration leads to xenon atom aggregation. Shown is the evolution of the aggregation that occurs at moderately short-range in which clusters of size $6-9$ Xe atoms are observed to form. Snapshots are at 100, 200, 600, and 1500 ps. Xenon atoms are in blue, uranium atoms in green and oxygen atoms in red. Note the increasing crystalline structure forming as the cluster forms.

\section{Model and Simulation Methodology}

Ideal fully stoichiometric $\mathrm{UO}_{2}$ assumes the fluorite-structure as a single crystal, where the cations are arranged in a face centered cubic structure and the anions are located at tetrahedral sites, creating a simple cubic sublattice within the unit cell. The system used in this study is a polycrystalline cell comprised of six columnar grains, whose orientation of atoms is rotated by 30 (degrees) with respect to one another. This allows for a fully periodic system to be modeled.

The initial stoichiometric supercell configuration was approximately $22 \AA \times 300 \AA \times 512 \AA$ in size that contained 244,182 atoms. It was modified to incorporate a supersaturation of point defects by introducing a $10 \%$ Schottky defect concentration. To create the defects, trivacancies in the form of neutral $\mathrm{UO}_{2}$ Schottky defects were randomly removed from the initial system. At approximately $25 \%$ of these trivacancy defects xenon atoms were randomly introduced, where each xenon atom occupied the space of 1-uranium and 2 adjacent oxygen atom vacancies.

The Yakub ${ }^{1}$ interaction potential is used for these simulations where the mathematical model that govern this interaction potential is given by

$$
V_{i j}\left(r_{i j}\right)=\frac{q_{i} q_{j}}{r_{i j}}+A_{i j} \exp \left(-r_{i j} / \rho_{i j}\right)-\frac{C_{i j}}{r_{i j}^{6}}+D_{i j}\left[e^{-2 \beta_{i j}\left(r_{i j}-r_{i j}^{0}\right)}-2 e^{-2 \beta_{i j}\left(r_{i j}-r_{i j}^{0}\right)}\right]
$$

The first term describes the point charge electrostatic interaction, the second and third terms describe the short-range repulsion and dispersion and the last describes the Morse-type interaction of the ions $i$ and $j$ with charges $q_{i}$ and $q_{j}$. The Yakub potential is able to predict the properties of both solid and molten $\mathrm{UO}_{2}$ and predicts a pre-melting transition to occur around $2500 \mathrm{~K}$. It also predicts point defect formation energy in agreement to high level ab initio caculations.

Simulations employed the well established DL_POLY computer code $3.0 .9^{2}$. The initial cell was equilibrated at $300 \mathrm{~K}$ using the NPT ensemble. The defects were then introduced randomly and Xe atoms were randomly added into the system and that system equilibrated 
using the NPT ensemble at $300 \mathrm{~K}$ for approximately $10 \mathrm{ps}$. The Berendsen barostat and thermostat (with relaxation constants of 0.1 and 1 ps respectively) were used and the electrostati. interactions were calculated using the particle mesh Ewald summation with a cut off radius of $10 \AA$. After room temperature equilibration, the temperature of the system was instantaneously raised to $2500 \mathrm{~K}$ employing the NVT (Berendsen) ensemble to simulate high temperature effects present during reactor operation.

Petascale computing resources were provided by the Environmental Molecular Science Laboratory at the Pacific Northwest National Laboratory.

This work was carried out in collaboration with Dr. Ram Devanathan at the Pacific Northwest National Laboratory (PNNL). The participating student, Ms. Emily Moore, had the opportunity to spend two summers at PNNL once as an undergraduate as part of the SULI program, and a second summer as a Materials Science and Engineering graduate student as part of the PNNL Condensed Matter Summer School both times under the mentoring of Dr. Devanathan. Ms. Moore earned her MS degree from the Materials Science and Engineering deparment in May of 2010 and is currently working towards her PhD degree from the Centre d'Etudes de Saclay working under the auspices of Dr. Constantin Meis of the National Institute of Nuclear Science and Technology - CEA in Gif-sur-Yvette, France.

\section{Publications:}

'Emily Moore, L. René Corrales and Ram Devanathan, "Xe Bubble Precoarsening in a Model $\mathrm{UO}_{2}$ Nuclear Fuel: Early Stage Molecular Dynamics Simulation", J. Comp. Mat. (submitted) 2010.

\section{Presentations:}

'Emily Moore, L. René Corrales and Ram Devanathan, "Molecular Dynamics Simulation of Xe Bubble Formation in a Model $\mathrm{UO}_{2}$ Nuclear Fuel", Materials Research Society Meeting, Boston Fall 2009, poster.

\section{REFERENCES}

${ }^{1}$ E. Yakub, C. Ronchi, D. Staicu, J. Nucl. Mater 389 (2009) 119

${ }^{2}$ W. Smith and T.R. Forester, J. Mol. Graphics 14, 136 (1996) 